\section{On Friedman equation, quadratic laws and the geometry of our universe}

\author{
S Kalimuthu*
}

2/394, Kanjampatti P.O, Pollachi Via, Tamil Nadu 642003, India

\begin{abstract}
Einstein's special and general relativity revolutionized physics. The predictions of general relativity are Strong Lensing, Weak Lensing, Microlensing, Black Holes, Relativistic Jets, A Gravitational Vortex, Gravitational Waves, The Sun Delaying Radio Signals, Proof from Orbiting Earth, Expansion of the universe. The density of the universe determines the geometry and fate of the universe. According to Freedman's equations of general relativity published in 1922 and 1924, the geometry of the universe may be closed, open and flat. It all depends upon the curvature of the universe also. Various results of Cosmic Microwave Background Radiation (CMBR), NASA's Wilkinson Microwave Anisotropy Probe (WMAP), and ESA's Planck spacecraft probes found that our universe is flat within a margin of $0.4 \%$ error. In this short work, by applying the laws of quadratic equations, we attempt to show that OUR UNIVERSE IS FLAT.
\end{abstract}

More Information

*Address for Correspondence: S Kalimuthu, 2/394, Kanjampatti P.O, Pollachi Via, Tamil Nadu 642003, India, Tel: +91 82205415 77; Email: owlskalimuthu@gmail.com

Submitted: August 28, 2020

Approved: June 24, 2021

Published: June 25, 2021

How to cite this article: Kalimuthu S Working process of steam turbine and establishment of start-up model. Int J Phys Res Appl. 2021; 4: 048-050.

DOI: 10.29328/journal.ijpra.1001041 ORCiD: orcid.org/0000-0001-7978-9013

Copyright: (c) 2021 Kalimuthu S. This is an open access article distributed under the Creative Commons Attribution License, which permits unrestricted use, distribution, and reproduction in any medium, provided the original work is properly cited.

Keywords: Freidman Equations; The fate of our Universe; MSC 08C99; PACS numbers: 98.70. Vc, 98.80.-k, 98.80.Es

\section{Check for updates}

OPEN ACCESS
The density parameter $\Omega$, the curvature parameter $\mathrm{k}$ and the Hubble parameter $\mathrm{H}$ are related as [7].

$$
(1-\Omega)=-\mathrm{kc}^{2} / \mathrm{H}^{2} \mathrm{R}^{2}
$$

If omega less than $1, k$ is - 1, If omega is equal to $1, k$ is zero, If omega greater than $1, k$ is +1 . If $k$ is -1 , the geometry of the universe is open, if it is greater than one, the shape of the universe is closed and the universe obeys Euclidean geometry if $k$ is equal to zero.

i.e if $\Omega=1$, the universe is Euclidean, if $\Omega=$ less than 1 the geometry of the universe is open, and if $\Omega=$ greater than 1 , the universe is closed.

\section{First method}

Squaring (1), $1+\Omega 2-2 \Omega-\mathrm{k}^{2} \mathrm{c}^{4} / \mathrm{H}^{4} \mathrm{R}^{4}=0$

According to the laws of quadratic equations, if Alpha and Beta are the roots, then,

The sum of the roots is given by, $\alpha+\beta=-b / a=2$

Since equation (5) is a cosmological one, the roots must be distinct and positive (1).

By assuming the above principle (2) in (1b) and applying, +1 for Alpha and Beta.

We get that LHS = RHS (3).
By assuming the above principle (2) in (1b) and applying, - 1/2 for Alpha and Beta.

$$
\text { We get }-1=2
$$

By assuming the above principle (2) in (1b) and applying, $1+\mathrm{x}$ for Alpha and Beta.

We get that $2+2 x=2$

Needless to say, both equations (4) and (5) are contradictions.

This implies that our assumptions that $\Omega$ is less than 1 and $\Omega$ is greater than 1 are not applicable (6).

Consequently we get that our application that $\Omega=1$ is acceptable (7).

If the discriminant is zero, then there is exactly one real root, $-\mathrm{b} / 2 \mathrm{a}$.

Here $\mathrm{b}=2$ and $\mathrm{a}=1$, So, $-\mathrm{b} / 2 \mathrm{a}=2 / 2=1$

As we have previously seen that is $\Omega=1$, then the geometry of our universe is FLAT.

\section{Second method}

Discriminant $\mathrm{B}^{2}-4 \mathrm{AC}$ 
A quadratic equation with real coefficients can have either one or two distinct real roots, or two distinct complex roots. In this case the discriminant determines the number and nature of the roots. There are three cases

If the discriminant is positive, then there are two distinct roots.

If the discriminant is zero, then there is exactly one real root, $-\mathrm{b} / 2 \mathrm{a}$.

If the discriminant is negative, then there are no real roots. Rather, there are two distinct (nonreal) complex roots.

$$
\begin{aligned}
& 1-\Omega=-\mathrm{kc}^{2} / \mathrm{H}^{2} \mathrm{R}^{2} \\
& 1+\Omega^{2}-2 \Omega-\mathrm{k}^{2} \mathrm{c}^{4} / \mathrm{H}^{4} \mathrm{R}^{4}=0
\end{aligned}
$$

The above equation is quadratic in $\Omega$.

\section{Case 1}

According to the laws of quadratic equations, if $\mathrm{B}^{2}-4 \mathrm{AC}=$ 0 , then the roots are real and distinct.

$$
\text { In (1a) } B=2, A=1 \text { and } C=-k^{2} c^{4} / H^{4} R^{4}
$$

Applying this in the above relation, we get that, $4-4+4$ $\mathrm{k}^{2} \mathrm{c}^{4} / \mathrm{H}^{4} \mathrm{R}^{4}=0$ i.e we obtain that $\mathrm{k}=0$.

We have already seen that if $\Omega=1$, then $\mathrm{k}=0$.

From this result, we may conclude that the roots of $\Omega$ may be distinct and real Result (A)

\section{Case 2}

Let us assume that in (1a), B2 - 4AC is greater than 0 , and let it be 1

Now, $B^{2}-4 A C=1$

In (1a) $B=2, A=1$ and $C=-k^{2} c^{4} / H^{4} R^{4}$

Applying this in the previous relation we get that, $=4-4+$ $4 \mathrm{k}^{2} \mathrm{c}^{4} / \mathrm{H}^{4} \mathrm{R}^{4} \quad=1$

Simplifying, $\mathrm{k}^{2} \mathrm{c}^{4} / \mathrm{H}^{4} \mathrm{R}^{4}=1 / 4$

Assuming the above relation in (1a) we obtain that, $3 / 4+\Omega^{2}-2 \Omega=0$

Multiplying throughout by $4,3+4 \Omega^{2}-8 \Omega=0$

$$
\begin{aligned}
& \text { Therefore, } \frac{\Omega=8 \text { plus or minus }(64-48)^{1 / 2}}{8} \\
& \text { i.e } \quad \Omega \quad=\frac{=4 \text { plus or minus }(16)^{1 / 2}}{4} \\
& \text { i.e } \quad \Omega \quad=\frac{=4 \text { plus or minus } 4}{4}
\end{aligned}
$$

$$
\text { i.e } \Omega \quad=1 \text { plus or minus } 1 \text { (Result B) }
$$

Choosing positive value and applying Result B in (1a) we get that, $1+4-4-\mathrm{k}^{2} \mathrm{c}^{4} / \mathrm{H}^{4} \mathrm{R}^{4}=0$

$$
\text { i.e } \quad 1=\mathrm{k}^{2} \mathrm{c}^{4} / \mathrm{H}^{4} \mathrm{R}^{4}
$$

Replacing $-\mathrm{k}^{2} \mathrm{c}^{4} / \mathrm{H}^{4} \mathrm{R}^{4}$ by 1 [ See above relation] in (1a) we have, $\Omega=2($ Result C )

Choosing negative value of Result B and applying in (1a) we get that, $1=\mathrm{k}^{2} \mathrm{c}^{4} / \mathrm{H}^{4} \mathrm{R}^{4}$

Assuming the above relation in $(1 \mathrm{a} 0$ we have, $\Omega=2$ (Result D)

Let us notice that results $\mathrm{C}$ and $\mathrm{D}$ are similar.

\section{Case 3}

Let us assume that $\mathrm{B}^{2}-4 \mathrm{AC}=$ less than zero $=-1$

Applying this in (1a) we have, $4-4+4 \mathrm{k}^{2} \mathrm{c}^{4} / \mathrm{H}^{4} \mathrm{R}^{4}=-1$

i.e $\mathrm{k}^{2} \mathrm{c}^{4} / \mathrm{H}^{4} \mathrm{R}^{4}=[-1 / 4]$

Taking root on both sides, $\mathrm{kc}^{2} / \mathrm{H}^{2} \mathrm{R}^{2}=\mathrm{i} / 2$

From this relation we obtain that $\mathrm{k}$ is imaginary (Result $\mathrm{E}$ )

Since $\mathrm{k}$ is the curvature parameter of our universe, it cannot be imaginary.

So, our case (3) is not acceptable (Result F)

\section{Discussion}

In the first method we have shown that the shape of our universe is Euclidean. In the second method, case 3 (Result F) is not acceptable. So, cases 1 and 2 are acceptable. The second method shows that the universe may be either closed or flat. But the first method proves that the t geometry of the universe is Euclidean/flat. The author has previously found several results in this topic [1-7].

"Finally, it could be that there's just enough matter for the Universe to have zero curvature. Angles of triangles add up to exactly 180 degrees and the Universe is infinite. If this is the case then it may be that matter can slow the rate of expansion, but it'll never be able to bring it down to zero. The Universe will also expire in a big freeze

Observations of the cosmic microwave background (the leftover radiation from the Big Bang) suggest that the Universe is indeed flat, or at least very nearly so. This would imply eternal expansion, at least if the assumptions above are true [https:// plus.maths.org/content/shape-and-fate-universe].

A brief analysis of previous experiments, observations and this author's mathematical formulations make us to arrive at a conclusion that the fate of our Universe is that it will expire in a big freeze. 


\section{References}

1. https://old.inspirehep.net/record/1795190

2. https://old.inspirehep.net/record/1806710

3. https://old.inspirehep.net/record/1826734
4. https://old.inspirehep.net/record/1833036
5. https://old.inspirehep.net/record/1833037
6. https://www.sciencepub.net/academia/aaj130421/
7. https://www.peertechzpublications.com/articles/AMP-3-115.pdf 